\title{
Bacterial Luciferases from Vibrio harveyi and Photobacterium leiognathi Demonstrate Different Conformational Stability as Detected by Time-Resolved Fluorescence Spectroscopy
}

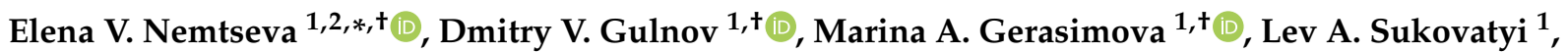 \\ Ludmila P. Burakova ${ }^{1,2}{ }^{\circledR}$, Natalya E. Karuzina ${ }^{1}$, Bogdan S. Melnik ${ }^{3}\left(\mathbb{D}\right.$ and Valentina A. Kratasyuk ${ }^{1,2}$ \\ 1 Siberian Federal University, 660041 Krasnoyarsk, Russia; dgulnov@sfu-kras.ru (D.V.G.); \\ marina_2506@mail.ru (M.A.G.); lsukovatyy@sfu-kras.ru (L.A.S.); burakoval@mail.ru (L.P.B.); \\ nkaruzina@sfu-kras.ru (N.E.K.); vkratasyuk@sfu-kras.ru (V.A.K.) \\ 2 Photobiology Laboratory, Institute of Biophysics SB RAS, 660036 Krasnoyarsk, Russia \\ 3 Institute of Protein Research, Russian Academy of Sciences, 142290 Pushchino, Russia; \\ bmelnik@phys.protres.ru \\ * Correspondence: enemtseva@sfu-kras.ru \\ + These authors contributed equally to this work.
}

check for updates

Citation: Nemtseva, E.V.; Gulnov, D.V.; Gerasimova, M.A.; Sukovatyi, L.A.; Burakova, L.P.; Karuzina, N.E.; Melnik, B.S.; Kratasyuk, V.A. Bacterial Luciferases from Vibrio harveyi and Photobacterium leiognathi Demonstrate Different Conformational Stability as Detected by Time-Resolved Fluorescence Spectroscopy. Int. J. Mol. Sci. 2021, 22, 10449. https://doi.org/ 10.3390/ijms221910449

Academic Editor: Konstantin K. Turoverov

Received: 30 August 2021

Accepted: 23 September 2021

Published: 28 September 2021

Publisher's Note: MDPI stays neutral with regard to jurisdictional claims in published maps and institutional affiliations.

Copyright: () 2021 by the authors. Licensee MDPI, Basel, Switzerland. This article is an open access article distributed under the terms and conditions of the Creative Commons Attribution (CC BY) license (https:// creativecommons.org/licenses/by/ $4.0 /)$.

\begin{abstract}
Detecting the folding/unfolding pathways of biological macromolecules is one of the urgent problems of molecular biophysics. The unfolding of bacterial luciferase from Vibrio harveyi is well-studied, unlike that of Photobacterium leiognathi, despite the fact that both of them are actively used as a reporter system. The aim of this study was to compare the conformational transitions of these luciferases from two different protein subfamilies during equilibrium unfolding with urea. Intrinsic steady-state and time-resolved fluorescence spectra and circular dichroism spectra were used to determine the stages of the protein unfolding. Molecular dynamics methods were applied to find the differences in the surroundings of tryptophans in both luciferases. We found that the unfolding pathway is the same for the studied luciferases. However, the results obtained indicate more stable tertiary and secondary structures of $P$. leiognathi luciferase as compared to enzyme from $V$. harveyi during the last stage of denaturation, including the unfolding of individual subunits. The distinctions in fluorescence of the two proteins are associated with differences in the structure of the C-terminal domain of $\alpha$-subunits, which causes different quenching of tryptophan emissions. The time-resolved fluorescence technique proved to be a more effective method for studying protein unfolding than steady-state methods.
\end{abstract}

Keywords: bacterial luciferase; urea-induced denaturation; time-resolved spectroscopy; conformational stability; FRET; tryptophan fluorescence; molecular dynamics; unfolding pathway

\section{Introduction}

The conformational stability of proteins and the methods of its evaluation have been the subject of intense interest for researchers from various fields, including molecular and cellular biophysics, biochemistry, food chemistry, biomedicine and many others. The disturbance of protein stability due to gene mutations or the influence of external physical and chemical factors can lead to the appearance of misfolded species or structural intermediates which cause various disease states in cells and tissues [1,2]. Fluorescence spectroscopy techniques are widely used to probe the conformational transitions of proteins [3]. The analysis of the time-resolved protein fluorescence has several advantages over other optical steady-state techniques; for example, fluorescence lifetimes do not depend on protein concentration, which is especially important for in vivo studies [4]. Meanwhile, there is a downside to the use of tryptophan residues as natural fluorophores to monitor the conformational states of proteins. Namely, it seems difficult to compare the unfolding pathways of two proteins bearing different numbers of tryptophans, which are located 
in different parts of the protein globule. An additional difficulty arises from the lack of understanding of the nature of the components of the tryptophan fluorescence lifetime. In the current study, we investigated whether time-resolved fluorescence can be used to detect differences in conformational stability under the urea-induced unfolding of two highly homologous multi-tryptophan proteins-bacterial luciferases. Molecular modelling methods were utilized to confirm that the observed differences are not caused by the distinct locations of tryptophans within the protein globules.

Luciferases catalyze specific reactions with the formation of a product in an electronically excited state; this product deactivates with the emission of light within the visible range of the spectrum. These chemical reactions ensure bioluminescence in living organisms, which serves various important functions. Bacterial luciferase catalyzes the oxidation of reduced flavin mononucleotides and long chain aliphatic aldehydes accompanied by the generation of blue-greenish light [5]. This enzymatic reaction is widely used as a reporter system in different fields, including molecular biology [6], environmental monitoring [7] and medical diagnostics [8].

Phylogenetic analysis of luciferase amino acid sequences have revealed that all known bacterial luciferases can be divided into two subfamilies [9], which are conditionally called "fast" and "slow" enzymes based on the kinetic peculiarities of their reactions [10]. Historically, during the last several decades, the bulk of research on bacterial bioluminescent reaction has been conducted using Vibrio harveyi luciferase, belonging to the "slow" enzymes. Those studies were concerned with the mechanism of catalysis [11], the structure [12,13], the folding and unfolding processes [14], the structure-function relationship [15], and other aspects. Meanwhile, the evident division of the luciferase sequences into two groups with high homology within each group suggests that the subfamilies could differ not only in their functional properties, but also in conformational stability. The published data do not allow us to confirm or refute this assumption, since luciferase unfolding has never been studied for at least two enzymes from different phylogenetic groups under the same conditions. In this work, we attempted to fill this gap by analyzing the conformational transitions of the "fast" P. leiognathi luciferase and the "slow" V. harveyi luciferase.

Structurally, bacterial luciferase is composed of two non-identical subunits $(\alpha$ and $\beta)$, both of which assume the $(\beta / \alpha)_{8}$ barrel fold [12]. Apart from the high similarity of the secondary and tertiary structures, the $\alpha$ - and $\beta$-subunits have a high sequence homology, especially at the $\mathrm{N}$-terminal, that reflects their possible origin from the same gene as a result of duplication [16]. However, the $\alpha$-subunit contains the active site and is larger due to the insertion of a mobile loop playing an important role in binding substrates and shielding reaction intermediates in the active site $[15,17]$. The dimerization of luciferase involves forming 22 hydrogen bonds between conserved amino acids of the subunits [12,18]. It is noteworthy that mutation of these amino acids leads not only to dissociation of the heterodimer, but in some cases to conformational changes in the $\alpha$-subunit (including the structure of the active site) $[13,19,20]$. Thorough study of the denaturation of $V$. harveyi luciferase with urea led to the conclusion about the four-state mechanism of unfolding, shown in Scheme 1 [20].

$$
(\alpha \beta)_{N} \leftrightarrow(\alpha \beta)_{I} \leftrightarrow \alpha_{I}+\beta_{I} \leftrightarrow \alpha_{U}+\beta_{U}
$$

Scheme 1. Four-state mechanism of $V$. harveyi luciferase unfolding with urea. $(\alpha \beta)_{N}$-luciferase heterodimer in the native state, $(\alpha \beta)_{I}$-inactive dimeric intermediate, $\alpha_{I}$ and $\beta_{I}$-dissociated subunits in the folded state, $\alpha_{U}$ and $\beta_{U}$-unfolded subunits.

The first intermediate $(\alpha \beta)_{I}$ was found to populate at about $2 \mathrm{M}$ of urea, while folded dissociated subunits $\alpha_{I}$ and $\beta_{I}$ populated at $3.5 \mathrm{M}$ of urea [14].

The aim of this study was to compare the conformational transitions of "fast" P. leiognathi and "slow" V. harveyi luciferases during equilibrium unfolding with urea. To detect the structural transitions of the proteins, we used optical techniques, including steadystate and time-resolved fluorescence spectroscopy and circular dichroism spectroscopy. In 
addition to information on the stability of the two homologous proteins, we attempted to clarify the question of whether the tryptophan fluorescence lifetime of bacterial luciferase carries new information about the unfolding pathway as compared to widely used steady-state methods. In previous studies, we established that the analysis of fluorescence lifetime allowed for the resolution of two distinct transitions of bovine carboanhydrase II during equilibrium unfolding with urea, which was impossible using other non-kinetic techniques [21]. One more issue that we endeavored to address is the dependence of conformational stability parameters obtained using fluorescence spectroscopy on the number of the tryptophan residues contained in the protein. The studied proteins have different numbers of tryptophans in their structures: P. leiognathi luciferase contains seven $(\alpha \mathrm{W} 40$, $\alpha \mathrm{W} 182, \alpha \mathrm{W} 194, \alpha \mathrm{W} 250, \alpha \mathrm{W} 277, \beta \mathrm{W} 182, \beta \mathrm{W} 194)$, while $V$. harveyi contains eight (the same plus $\alpha$ W131).

\section{Results}

\subsection{Fluorescence Lifetime Change during Urea-Induced Unfolding of Luciferases}

We measured the fluorescence decays under excitation of $296 \mathrm{~nm}$ for $V$. harveyi and $P$. leiognathi luciferases in buffer and after incubation at various urea concentrations. The global analysis of the decays in a wide emission range $(305-410 \mathrm{~nm})$ made it possible to determine not only lifetimes, but also their contributions to the overall fluorescence, which allowed the decay-associated spectra of the proteins to be obtained. The deconvolution procedure revealed 3 lifetime components: 4.5-6.0 ns $\left(\tau_{1}\right), 1.7-2.1 \mathrm{~ns}\left(\tau_{2}\right)$, and 0.03-0.2 ns $\left(\tau_{3}\right)$ for each sample. The components $\tau_{1}$ and $\tau_{2}$ together made the major contribution to the protein fluorescence of all samples (about $90 \%$ ), and their values could be reliably resolved by the instrument, unlike the value of $\tau_{3}$. That is why the $\tau_{3}$ change during protein unfolding is not discussed in the current investigation. The dependence of $\tau_{1}$ and $\tau_{2}$ on the urea concentration is shown in Figure 1A, demonstrating a similar change of the two lifetime components for each luciferase. At $>3.5-4 \mathrm{M}$ of urea, $\tau_{1}$ and $\tau_{2}$ decrease for both proteins. However, at low urea concentrations, contrasting patterns were revealed for both studied luciferases: for $V$. harveyi, an increase in the lifetimes was observed up to $1.5 \mathrm{M}$, whereas for P. leiognathi luciferase, a decrease in the lifetimes was detected in the concentration range of $0-2 \mathrm{M}$. On the whole, the change in fluorescence lifetimes with increasing urea concentrations reflects two structural transitions for each luciferase. From the previous study of $V$. harveyi luciferase unfolding, it was concluded that two intermediates of this protein populated at about $2 \mathrm{M}$ and $3.5 \mathrm{M}$ [20] of urea. A comparison of the spectral properties of these intermediates, as well as the native and unfolded states of two luciferases, are presented in Figure 1B-E. One can see that: (i) $V$. harveyi luciferase demonstrates a higher intensity of fluorescence at the same concentration of urea (Figure 1B-E), which was as expected due to the extra tryptophan residue in its structure as compared to $P$. leiognathi luciferase; (ii) for $V$. harveyi luciferase, the fluorescence lifetime increase in the range $0-2 \mathrm{M}$ of urea is accompanied by a fluorescence intensity increase (Figure 1B,C); (iii) the two lifetime components $\tau_{1}$ and $\tau_{2}$ have similar spectral fractions in protein fluorescence at all urea concentrations. It is noteworthy that the spectral fractions of the two fluorescence lifetimes were found to remain nearly the same at all studied urea concentrations (Figure S1). 
A

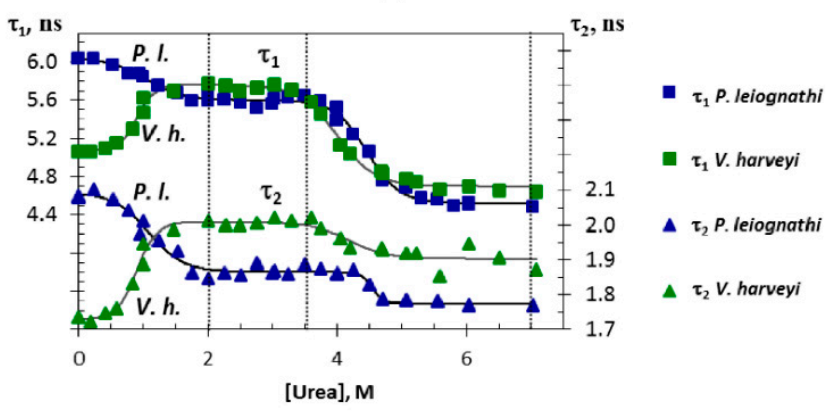

B

C

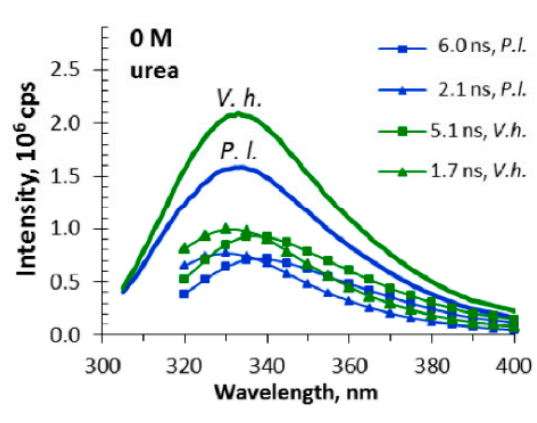

D

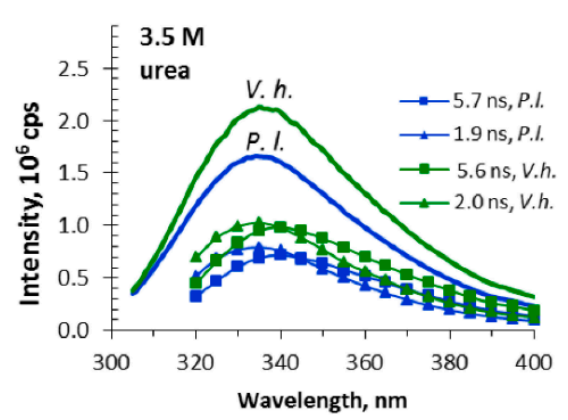

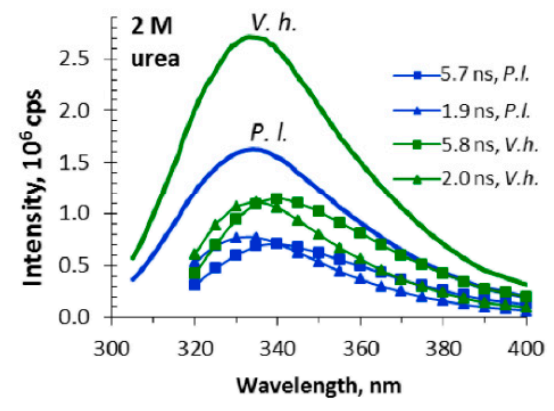

$\mathrm{E}$

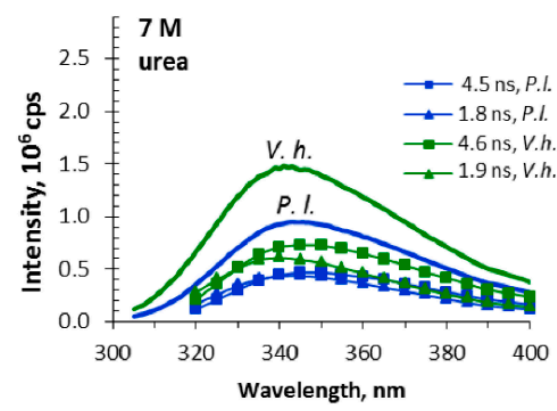

Figure 1. Dependence of the fluorescence lifetime components $\tau_{1}$ (squares) and $\tau_{2}$ (triangles) on urea concentration for P. leiognathi (blue) and V. harveyi (green) luciferases (A), and the steady-state fluorescence spectra of native $(\mathbf{B})$, intermediate $(\mathbf{C}, \mathbf{D})$ and unfolded $(\mathbf{E})$ states of luciferases (excitation at $296 \mathrm{~nm}$, protein concentration is $1.7 \mu \mathrm{M})$. In panel (A), vertical dotted lines refer to the urea concentrations at which the fluorescence spectra are shown in panels $(\mathbf{C}-\mathbf{E})$; solid lines represent fitting to a double Boltzmann function. By marked lines in panels (B-E) the decay-associated spectra based on Equation (2) are shown for the lifetime components.

Additionally, we obtained the transition curves of luciferases using the other optical parameters: the molar ellipticity at $222 \mathrm{~nm}$ in the circular dichroism spectra $\left(\Theta_{222}\right)$, and the ratio of intensities at 320 and $360 \mathrm{~nm}$ in the steady-state fluorescence spectra $\left(I_{320} / I_{360}\right)$. A comparison of the parameters obtained by different methods is shown in Figure 2. One can see that variation of $\Theta_{222}$ reveals two transitions similar to those detected by the fluorescence lifetime component $\tau_{1}$, whereas the change of $\mathrm{I}_{320} / \mathrm{I}_{360}$ is not so pronounced for the urea concentrations $<2 \mathrm{M}$, especially for $V$. harveyi luciferase. The entire transition curves were fitted by the double Boltzmann function (see the Section 4) (Figure 2). 
A

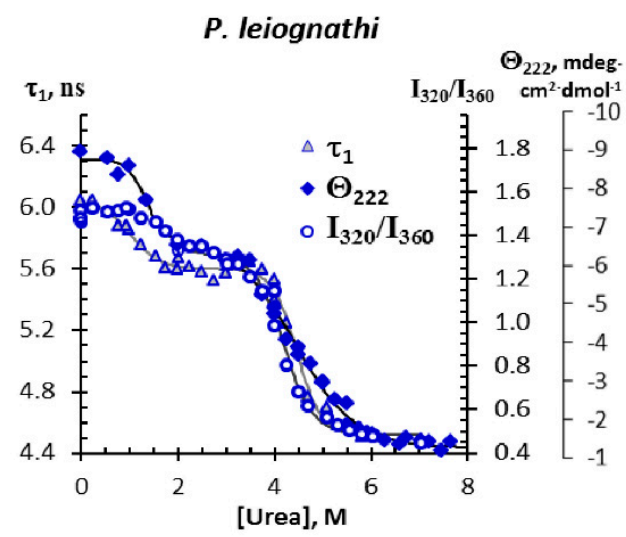

B

V. harveyi

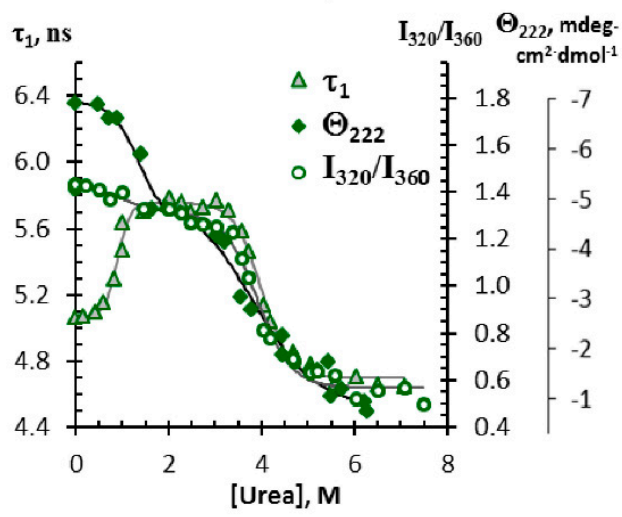

Figure 2. Comparison of the optical parameter changes in equilibrium unfolding of P. leiognathi (A) and V. harveyi (B) luciferases obtained by different methods: the ratio $\mathrm{I}_{320} / \mathrm{I}_{360}(\bigcirc)$, molar ellipticity at $222 \mathrm{~nm}, \Theta_{222},(\diamond)$ and the fluorescence lifetime component $\tau_{1}(\triangle)$. Solid lines represent fitting to a double Boltzmann function.

The fitted transitions detected by different methods allowed thermodynamic parameters to be calculated and, hence, the structural stability of the two luciferases to be compared (Table 1).

Table 1. Thermodynamic parameters of two stages of luciferase unfolding with urea. The parameters which differ for the two luciferases are in bold.

\begin{tabular}{|c|c|c|c|c|c|c|c|c|c|}
\hline \multirow{2}{*}{ № } & \multirow{2}{*}{ Method } & \multicolumn{2}{|c|}{ Fraction of Transition ${ }^{\#}$} & \multicolumn{2}{|c|}{$\Delta \mathrm{G}_{\mathrm{H} 2 \mathrm{O}}, \mathrm{kcal} / \mathrm{mol}^{*}$} & \multicolumn{2}{|c|}{$\mathrm{m}_{\mathrm{U}}, \mathrm{kcal} / \mathrm{M} / \mathrm{mol}^{*}$} & \multicolumn{2}{|c|}{ [Urea] $_{50 \%}, \mathbf{M}^{*}$} \\
\hline & & P. $l$. & V. $h$. & P. $l$. & V. $h$. & P. $l$. & V. $h$. & P. $l$. & V. $h$. \\
\hline \multirow{2}{*}{1} & \multirow{2}{*}{$\mathrm{I}_{320} / \mathrm{I}_{360}$} & 0.18 & 0.15 & $5.2 \pm 2.3$ & $1.6 \pm 2.0$ & $2.8 \pm 1.3$ & $1.5 \pm 0.3$ & $\mathbf{1 . 8} \pm 0.1$ & $\mathbf{1 . 0} \pm 0.4$ \\
\hline & & 0.82 & 0.85 & $8.4 \pm 0.7$ & $6.6 \pm 0.6$ & $2.0 \pm 0.2$ & $1.7 \pm 0.2$ & $4.2 \pm 0.1$ & $3.9 \pm 0.1$ \\
\hline \multirow{2}{*}{2} & \multirow{2}{*}{$\Theta_{222}$} & 0.27 & 0.34 & $4.2 \pm 0.9$ & $4.1 \pm 0.8$ & $3.2 \pm 0.9$ & $3.0 \pm 1.2$ & $1.3 \pm 0.1$ & $1.4 \pm 0.2$ \\
\hline & & 0.73 & 0.66 & $4.3 \pm 0.6$ & $3.8 \pm 0.6$ & $1.0 \pm 0.2$ & $1.0 \pm 0.3$ & $4.4 \pm 0.1$ & $3.7 \pm 0.2$ \\
\hline \multirow{2}{*}{3} & \multirow[b]{2}{*}{$\tau_{1}$} & 0.30 & 0.39 & $2.1 \pm 0.3$ & $4.0 \pm 0.6$ & $2.0 \pm 0.3$ & $4.4 \pm 0.7$ & $1.0 \pm 0.1$ & $0.9 \pm 0.1$ \\
\hline & & 0.70 & 0.61 & $\mathbf{1 0 . 8} \pm 0.9$ & $8.2 \pm 0.7$ & $2.4 \pm 0.2$ & $2.0 \pm 0.2$ & $4.5 \pm 0.1$ & $4.0 \pm 0.1$ \\
\hline \multirow{2}{*}{4} & \multirow{2}{*}{$\tau_{2}$} & 0.70 & 0.73 & $2.5 \pm 0.4$ & $3.5 \pm 0.8$ & $2.3 \pm 0.3$ & $3.9 \pm 0.9$ & $1.1 \pm 0.1$ & $0.9 \pm 0.1$ \\
\hline & & 0.30 & 0.27 & $11.0 \pm 4.3$ & $8.5 \pm 4.2$ & $2.4 \pm 0.9$ & $2.0 \pm 1.0$ & $4.6 \pm 0.2$ & $4.2 \pm 0.2$ \\
\hline
\end{tabular}

" Calculated as the ratio of parameter change in the individual transition to the total parameter change upon denaturation. ${ }^{*}$ Calculated from the fitting curves in Figures $1 \mathrm{~A}$ and 2.

The observed difference in fluorescence characteristics of the two proteins during unfolding could have been caused by the distinguished location of tryptophan residues in the protein globule and/or the different microenvironment of these fluorophores. To check this relation, we analyzed the structural and dynamic properties of the two luciferases using the molecular modeling technique.

\subsection{Tryptophans of the Bacterial Luciferases as Fluorescent Reporters}

To compare the position of tryptophan residues and the properties of their microenvironment in the two luciferases, molecular dynamics simulations of the proteins were performed for $40 \mathrm{~ns}$. The tertiary structure of P. leiognathi luciferase was modeled for the first time (see details in the Section 4). We found that seven tryptophans of P. leiognathi luciferase are located in approximately the same position as compared to $V$. harveyi luciferase, in spite of a slight difference in the total sequence lengths of the proteins. The relative positions of those tryptophans in the luciferases after the tertiary structure alignment are presented in Figure S2. Instead of $\alpha \mathrm{W} 131$ presented in V. harveyi luciferase, the P. leiognathi structure contains a phenylalanine residue in the same position (Figure S2). The position 
of $\alpha \mathrm{W} 277$ (located in the mobile loop of the luciferases) revealed the most prominent difference between the proteins (Figure S2).

For each tryptophan, the microenvironment properties, which are known to influence the fluorescence characteristics of this residue, were determined [22]. In particular, the analyzed parameters include the total number of protein atoms within $7 \AA$ of any tryptophan atom $\left(\mathrm{N}_{\Sigma}\right)$ and the number of polar atoms among them $\left(\mathrm{N}_{\mathrm{pol}}\right)$, the solvent-accessible surface area (SASA), and others. All the calculated characteristics are summarized in Table 2.

Table 2. Microenvironment characteristics of tryptophan residues in V. harveyi (V.h.) and P. leiognathi (P.l.) luciferase structures in their native state. The most remarkable differences between the proteins are in bold.

\begin{tabular}{|c|c|c|c|c|c|c|c|c|c|c|c|c|c|}
\hline \multirow{2}{*}{\multicolumn{2}{|c|}{ Tryptophan $\downarrow$}} & \multicolumn{10}{|c|}{ Characteristics of Microenvironment in $7 \AA$ * \& } & \multirow{2}{*}{\multicolumn{2}{|c|}{ SASA, $\AA^{2}(\%)^{\#}$}} \\
\hline & & \multicolumn{2}{|c|}{$\mathrm{N}_{\Sigma}, \mathrm{pcs}$} & \multicolumn{2}{|c|}{$\mathrm{N}_{\text {pol }}$, pcs, $(\%) \stackrel{\text { \& }}{ }$} & \multicolumn{2}{|c|}{$\mathbf{N}_{\mathrm{sec}}\left(\mathbf{N}_{\alpha}+\mathbf{N}_{\beta}\right), \mathrm{pcs},(\%) \&$} & \multicolumn{2}{|c|}{$\begin{array}{c}\mathrm{N}_{\mathrm{C}-\text { term, }} \mathrm{pcs}, \\
(\%)\end{array}$} & \multicolumn{2}{|c|}{$\begin{array}{c}\mathrm{N}_{\beta \text {-sub, pcs, }} \\
(\%) \&\end{array}$} & & \\
\hline Subunit & Position & P.l. & V.h. & P.l. & V.h. & P.l. & V.h. & P.l. & V.h. & P.l. & V.h. & P.l. & V.h. \\
\hline$\alpha$ & 40 & 159 & 177 & $\begin{array}{c}64 \\
(40)\end{array}$ & $\begin{array}{c}63 \\
(36)\end{array}$ & $\begin{array}{c}159 \\
(5+154) \\
(100)\end{array}$ & $\begin{array}{c}170 \\
(0+170) \\
(96)\end{array}$ & $\begin{array}{l}14 \\
(9)\end{array}$ & $\begin{array}{c}18 \\
(10)\end{array}$ & 0 & 0 & $\begin{array}{l}14.8 \pm 7.9 \\
(5.6 \pm 3.0)\end{array}$ & $\begin{array}{c}8.6 \pm 6.2 \\
(3.2 \pm 2.4)\end{array}$ \\
\hline$\alpha$ & 131 & - & 162 & - & $\begin{array}{c}59 \\
(36)\end{array}$ & - & $\begin{array}{c}99(84+15) \\
(61)\end{array}$ & - & $\begin{array}{l}12 \\
(7)\end{array}$ & - & 0 & - & $\begin{array}{c}7.2 \pm 5.9 \\
(2.7 \pm 2.2)\end{array}$ \\
\hline$\alpha$ & 182 & 135 & 148 & $\begin{array}{c}54 \\
(40)\end{array}$ & $\begin{array}{c}56 \\
(38)\end{array}$ & $\begin{array}{c}121 \\
(107+14) \\
(90)\end{array}$ & $\begin{array}{c}130 \\
(111+19) \\
(88)\end{array}$ & 0 & 0 & 0 & 0 & $\begin{array}{c}25.4 \pm 13.1 \\
(9.6 \pm 5.0)\end{array}$ & $\begin{array}{c}33.0 \pm 8.3 \\
(12.5 \pm 3.2)\end{array}$ \\
\hline$\alpha$ & 194 & 127 & 141 & $\begin{array}{l}39 \\
(31)\end{array}$ & $\begin{array}{c}44 \\
(31)\end{array}$ & $\begin{array}{c}78(34+44) \\
(61)\end{array}$ & $\begin{array}{c}84(36+48) \\
(60)\end{array}$ & $\begin{array}{c}50 \\
(39)\end{array}$ & $\begin{array}{c}60 \\
(43)\end{array}$ & 0 & 0 & $\begin{array}{l}19.3 \pm 7.9 \\
(7.3 \pm 3.0)\end{array}$ & $\begin{array}{l}20.8 \pm 7.9 \\
(7.9 \pm 3.0)\end{array}$ \\
\hline$\alpha$ & 250 & 121 & 151 & $\begin{array}{l}33 \\
(27)\end{array}$ & $\begin{array}{c}48 \\
(32)\end{array}$ & $\begin{array}{c}79(79+0) \\
(65)\end{array}$ & $\begin{array}{c}83(82+1) \\
(55)\end{array}$ & $\begin{array}{c}93 \\
(77)\end{array}$ & $\begin{array}{l}106 \\
(70)\end{array}$ & 0 & 0 & $\begin{array}{l}39.8 \pm 11.7 \\
(15.1 \pm 4.4)\end{array}$ & $\begin{array}{l}34.3 \pm 13.6 \\
(13.0 \pm 5.1)\end{array}$ \\
\hline$\alpha$ & 277 & 93 & 121 & $\begin{array}{c}39 \\
(42)\end{array}$ & $\begin{array}{c}52 \\
(43)\end{array}$ & $\begin{array}{c}67(0+67) \\
(72)\end{array}$ & $\begin{array}{c}20(20+0) \\
(17)\end{array}$ & $\begin{array}{c}63 \\
(68)\end{array}$ & $\begin{array}{c}64 \\
(53)\end{array}$ & $\begin{array}{c}28 \\
(30)\end{array}$ & 0 & $\begin{array}{l}114.2 \pm 22.8 \\
(43.3 \pm 8.6)\end{array}$ & $\begin{aligned} 99.4 & \pm 26.8 \\
(37.7 & \pm 10.2)\end{aligned}$ \\
\hline$\beta$ & 182 & 146 & 148 & $\begin{array}{c}48 \\
(32)\end{array}$ & $\begin{array}{c}47 \\
(32)\end{array}$ & $\begin{array}{c}118 \\
(101+17) \\
(81)\end{array}$ & $\begin{array}{c}132 \\
(111+21) \\
(89)\end{array}$ & 0 & 0 & $\mathrm{~N} / \mathrm{A}$ & $\mathrm{N} / \mathrm{A}$ & $\begin{array}{c}25.7 \pm 10.3 \\
(9.7 \pm 3.9)\end{array}$ & $\begin{array}{l}22.6 \pm 9.1 \\
(8.6 \pm 3.5)\end{array}$ \\
\hline$\beta$ & 194 & 145 & 152 & $\begin{array}{c}41 \\
(28)\end{array}$ & $\begin{array}{c}44 \\
(29)\end{array}$ & $\begin{array}{c}87(42+45) \\
(60)\end{array}$ & $\begin{array}{c}92(38+54) \\
(61)\end{array}$ & 0 & 0 & $\mathrm{~N} / \mathrm{A}$ & $\mathrm{N} / \mathrm{A}$ & $\begin{array}{l}17.8 \pm 6.5 \\
(6.7 \pm 2.5)\end{array}$ & $\begin{array}{c}6.1 \pm 5.1 \\
(2.3 \pm 1.9)\end{array}$ \\
\hline
\end{tabular}

${ }^{*} \mathrm{~N}_{\Sigma}$-total number of protein atoms; $\mathrm{N}_{\text {pol }}$-number of polar atoms; $\mathrm{N}_{\mathrm{sec}}\left(\mathrm{N}_{\alpha}+\mathrm{N}_{\beta}\right)$-number of the atoms included in secondary

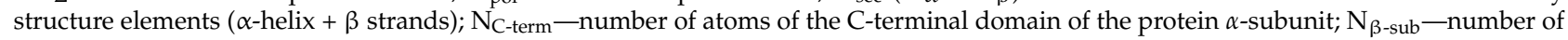
atoms of the protein $\beta$-subunit; SASA—solvent-accessible surface area (average value during MD simulation \pm standard deviation); ${ }^{*}$ in parentheses, the percentage of the corresponding atoms from the total number of atoms $\left(\mathrm{N}_{\Sigma}\right)$ is shown; ${ }^{\#}$ in parentheses, the percentage of the solvent-accessible surface area for the analyzed tryptophan residue from the maximal SASA for tryptophan in a protein is shown.

Thus, a packing density of the tryptophans (quantified by $\mathrm{N}_{\Sigma}$ ) is higher in $V$. harveyi luciferase, and the most buried ones are $\alpha \mathrm{W} 250$ and $\alpha \mathrm{W} 277$. However, the apparent polarity of the tryptophan microenvironments (as indicated by $\mathrm{N}_{\mathrm{pol}}$ ) does not differ significantly between the two luciferases.

The majority of the tryptophan residues in the luciferases demonstrate a small solventaccessible surface area ( $0-17 \%$ from the total surface area), i.e., the residues are buried in the protein globule. The exceptions are the $\alpha \mathrm{W} 277$ of both proteins, with about $40 \%$ of the surface area exposed to the solvent (Table 2). It is noteworthy that $\alpha \mathrm{W} 277$ also has the most flexible microenvironment in the studied structures, because a significant variation of its SASA values was observed during molecular dynamics simulations.

The first step of luciferase unfolding includes denaturation of the C-terminal domain of the protein (236-355 amino acid residues) [23]. To estimate the sensitivity of tryptophan fluorescence at this step, the number of the luciferase $\mathrm{C}$-terminal domain atoms neighboring each tryptophan was also counted $\left(\mathrm{N}_{\mathrm{C} \text {-term }}\right.$, Table 2). The highest value of $\mathrm{N}_{\mathrm{C} \text {-term }}$ was found for $\alpha \mathrm{W} 194$ and $\alpha \mathrm{W} 277$. Similarly, it was revealed that changes in the fluorescence spectra of any tryptophan (except for $\alpha \mathrm{W} 277$ of $V$. harveyi) can be seen in response to the secondary structure disruption, because their microenvironment mainly consists of the amino acids incorporated into $\alpha$-helixes or $\beta$-strands ( $>54 \%$ of atoms) $\left(\mathrm{N}_{\mathrm{sec}}\right.$, Table 2$)$.

The second stage of luciferase unfolding involves subunit dissociation. Among all tryptophans, only $\alpha \mathrm{W} 277$ of P. leiognathi luciferase was found to have the atoms of a $\beta$ subunit in its microenvironment within $7 \AA$ distance from the side chain atoms $\left(\mathrm{N}_{\beta \text {-sub }}\right.$, Table 2). 
The Förster resonance energy transfer (FRET) between nearby tryptophans can also have a strong effect on the protein fluorescence [24]; therefore, we performed a search for potential donor-acceptor pairs among the tryptophans of the luciferases. From distances $R$, it was concluded that FRET can occur only between $\alpha \mathrm{W} 194$ and $\alpha \mathrm{W} 250$. The aligned position of these tryptophan pairs in the two luciferases is presented in Figure 3A. Apparently, the donor-acceptor orientation is not identical for the two proteins. Using atom coordinates, we calculated the orientation factor $\kappa^{2}$ from Equation (5) and the FRET efficiency $E$ from Equation (4) between ${ }^{1} \mathrm{~L}_{\mathrm{a}}$ states of the tryptophans presented in Figure 3A. As a result, the value of $\kappa^{2}$ was 0.32 and 0.82 , and the efficiency was $86 \%$ and $82 \%$ for luciferases from $P$. leiognathi and $V$. harveyi, correspondingly. Notably, the observed less favorable orientation of the $V$. harveyi donor-acceptor pair is compensated by a shorter distance between the tryptophans. Taking into account the intrinsic dynamics of the proteins, we analyzed the distribution of the distances between $\alpha \mathrm{W} 194$ and $\alpha \mathrm{W} 250$ during molecular dynamics simulations (Figure 3B). One can see that $V$. harveyi luciferase demonstrates less structural mobility: most of the time its tryptophans are separated by the intervals of 5.5-6.5 $\AA$, whereas in P. leiognathi luciferase, the donor-acceptor distance varies in a wider range of 5.0-8.5 $\AA$. This finding correlates with the higher packing density of $\alpha \mathrm{W} 250$ in $V$. harveyi luciferase $\left(\mathrm{N}_{\Sigma}\right.$, Table 2$)$, which could have been preventing sidechain mobility. The observed distances for both proteins are shorter than the Förster radius for the Trp-Trp pair (7.8 $\AA$ ), what means that the FRET efficiency could have been higher than 50\% (Figure 3B). However, in the study of the mutants of the Photorhabdus luminescens luciferase (a close homologue of $V$. harveyi luciferase), it was concluded that for the donor-acceptor pair $\alpha$ W194- $\alpha$ W250, the range of FRET is restricted to a few angstroms. [25]. In general, our analysis indicates that the resonance energy transfer between $\alpha \mathrm{W} 194$ and $\alpha \mathrm{W} 250$ could have a stronger effect on the intrinsic fluorescence of $V$. harveyi luciferase than on that of $P$. leiognathi luciferase because of a shorter mean distance and lower protein dynamics. It should be noted that such short distances between tryptophans also do not allow us to exclude other mechanisms of energy transfer, such as electron transfer or charge transfer, which begin to be observed at $<10 \AA$ distances [26].

A

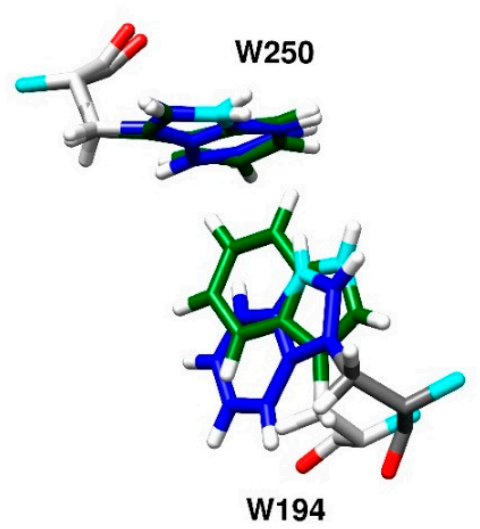

B

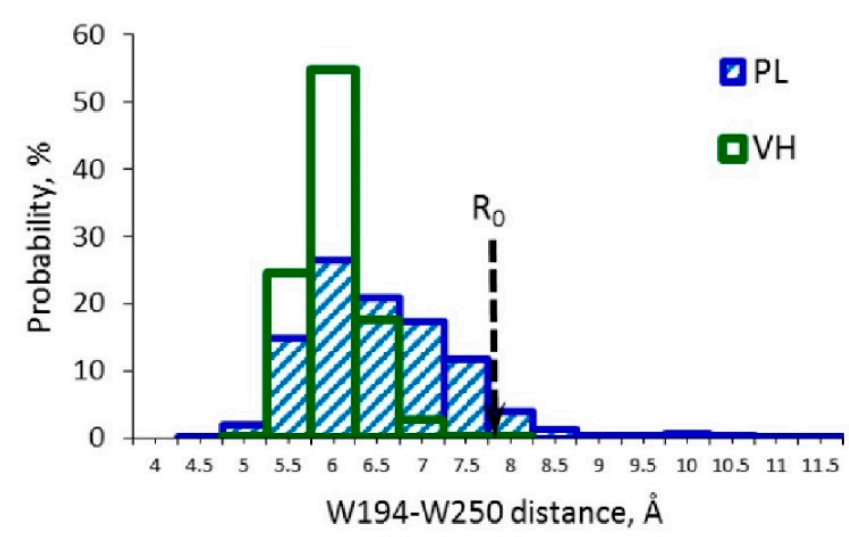

Figure 3. (A) Relative position of $\alpha \mathrm{W} 250$ (top) and $\alpha \mathrm{W} 194$ (bottom) in the structures of P. leiognathi (blue) and V. harveyi (green) luciferases. The protein structures after molecular dynamics simulations were aligned so that the positions of $\alpha \mathrm{W} 250$ coincided. The corresponding FRET efficiencies between ${ }^{1}$ La states are $86 \%$ (P. leiognathi) and $82 \%$ (V. harveyi). (B) The distribution of distances between $\alpha \mathrm{W} 250$ and $\alpha \mathrm{W} 194$ during molecular dynamics simulations of luciferases $P$. leiognathi (blue) and $V$. harveyi (green). The black arrow indicates the Förster radius $\left(\mathrm{R}_{0}\right)$ for FRET between the tryptophans [26].

An additional mechanism of change in the fluorescence intensity and the lifetime of tryptophans in a protein is quenching by the neighboring atoms of both amino acid side chains and the protein backbone chain [27]. Careful examination of the atoms surrounding the tryptophans revealed three differences between the luciferases: (i) the distance between 
the $\mathrm{C}_{\varepsilon 3}$ atom and the carbonyl group of $\alpha \mathrm{W} 182$ in the $V$. harveyi protein is shorter than in the P. leiognathi protein (3.6 and $4.5 \AA$, respectively); (ii) $\alpha \mathrm{D} 120$, known for its ability to quench tryptophan fluorescence with its side chain, is located $4 \AA$ away from $\alpha \mathrm{W} 277$ in $V$. harveyi, whereas in P. leiognathi luciferase all potential quenchers of $\alpha \mathrm{W} 277$ are at distances $>5.5 \AA$; (iii) the $\alpha \mathrm{W} 131$ of $V$. harveyi, which has no analogue in the P. leiognathi structure, resides near the sulfur atom of $\alpha \mathrm{C} 130(4.4 \AA)$, and its fluorescence can be also quenched. Notably, for Photorhabdus luminescens luciferase, the possibility of the $\alpha$ W182 fluorescence being quenched by the protein groups was revealed earlier [25]. Thus, a more pronounced effect of quenching on the tryptophan fluorescence could be expected in $V$. harveyi luciferase than in P. leiognathi luciferase.

\section{Discussion}

\subsection{The Origin of the Difference in Tryptophan Fluorescence of the Two Luciferases}

This study was aimed at comparing the structural stability of two highly homologous proteins-luciferases from bacteria P. leiognathi and $V$. harveyi-during urea-induced unfolding. For this, a detailed analysis of fluorescence properties, including the time-resolved properties, was first performed for the two luciferases after incubation at various urea concentrations. This study allowed us to identify features that differ for the proteins under consideration, despite the great similarities of their structures.

First, $V$. harveyi luciferase in its native state demonstrates a higher fluorescence intensity per one protein molecule, but a shorter fluorescence lifetime, than P. leiognathi luciferase (Figure 1B). Generally, a decrease in the fluorescence lifetime of a fluorophore indicates enhancement of the nonradiative pathways of the relaxation of the excitation energy (i.e., electron/proton/energy transfer, internal conversion and others) and correlates with a lower fluorescence quantum yield [27]. For tryptophan fluorescence in proteins, the following mechanisms of quenching are recognized [28]: (a) proton transfer from nearby charged amino groups, which is effective from lysine and tyrosine side chains; (b) electron transfer quenching by disulfides and amides or protonated carboxyl groups in which cysteine, histidine, glutamine, asparagine, as well as aspartic and glutamic acids, could take part; (c) electron transfer quenching by peptide bonds in the protein backbone; and (d) resonance energy transfer among the tryptophan residues.

We obtained data indicating that the shorter $\tau_{1}$ in the native state of $V$. harveyi luciferase compared to that of $P$. leiognathi luciferase $(5.1 \mathrm{vs.} 6.0 \mathrm{~ns})$ can be the result of a more effective FRET between $\alpha \mathrm{W} 194$ and $\alpha \mathrm{W} 250$ (mechanism (d)), facilitated by a closer spacing between these tryptophans (Figure 3 ) and by a higher packing density $\left(\mathrm{N}_{\Sigma}\right.$, Table 2). Additionally, examination of the tryptophan microenvironments revealed that in $V$. harveyi luciferase, the fluorescence of $\alpha \mathrm{W} 182$ can be quenched by its carbonyl group (mechanism (c)) and the fluorescence of $\alpha$ W277 can be quenched by $\alpha$ D120 (mechanism (b)), which is not the case for the P. leiognathi enzyme. Additionally, the presence of one more tryptophan $(\alpha \mathrm{W} 131)$ in the structure of the $V$. harveyi protein probably compensates for the loss of intensity due to fluorescence quenching, which explains the contradiction between the lifetimes and the intensities observed for the luciferases in their native state (Figure 1B). The identification of more precise mechanisms seems difficult in the scope of the current work because mutations of the tryptophan residues are required to estimate their contribution to the intensity and the lifetime components of the protein fluorescence.

Second, the fluorescence lifetimes of the two luciferases change in opposite ways during the first stage of denaturation (at $<2 \mathrm{M}$ of urea): for $V$. harveyi luciferase, $\tau_{1}$ and $\tau_{2}$ show an increase, while for the P. leiognathi enzyme, the lifetime components decrease (Figure 1A-C). This stage includes the unfolding of the C-terminal domain of the luciferase $\alpha$-subunit (236-355 a.r.) that was shown in different studies [23]. Thus, the increase of fluorescence intensity and the lifetimes $\tau_{1}$ and $\tau_{2}$ of $V$. harveyi luciferase at low urea concentrations could be the result of neutralizing the quenching effects of the microenvironment on the tryptophans discussed above. Indeed, $\alpha \mathrm{W} 277$ and $\alpha \mathrm{W} 250$ themselves are localized on the C-terminal domain, and in close proximity to $\alpha \mathrm{W} 194,43 \%$ of all atoms belong to 
this domain $\left(\mathrm{N}_{\mathrm{C} \text {-term }}\right.$, Table 2$)$. In turn, the small decrease of $\tau_{1}$ and $\tau_{2}$ of P. leiognathi luciferase during the first stage of unfolding can originate from the disordering of the $\alpha$ W277 microenvironment. According to the analysis, this tryptophan is located among the residues included in the $\beta$-strands $\left(\mathrm{N}_{\mathrm{sec}}\right.$, Table 2$)$, which could cause a higher quantum yield due to more ordered and less mobile surroundings. In this case, the unfolding of the C-terminal domain would result in a decrease of the fluorescence lifetime. It is noteworthy that the two luciferases in the intermediate state $(\alpha \beta)_{I}$ (at about $2 \mathrm{M}$ of urea) acquire almost identical fluorescence properties (Figure 1A,C). This means that all the differences observed in the native state are associated with the structural features of the C-terminal domain of the two luciferases. After the unfolding of this domain, the luciferases become almost indistinguishable by fluorescence spectroscopy.

The next denaturation stage is the subunit dissociation. This does not influence the optical characteristics of either of the luciferases, which is evident from a slight change in various parameters in the range of 2-3.5 M urea (Figures 1A,C,D and 2). This result is not surprising, since all the tryptophans, with the exception of $\alpha \mathrm{W} 277$ of P. leiognathi luciferase, are located far from the intersubunit interface $\left(\mathrm{N}_{\beta}\right.$, Table 2$)$.

The last stage of denaturation is the unfolding of the subunits. This occurs in the same way for the two proteins (Figures $1 \mathrm{~A}, \mathrm{D}, \mathrm{E}$ and 2) with the only difference seen in the fluorescence intensity, which is associated with the presence of the additional $\alpha \mathrm{W} 131$ in $V$. harveyi luciferase.

Probably due to the unidirectional change of the lifetimes $\tau_{1}$ and $\tau_{2}$ during denaturation, their relative contributions to the protein fluorescence do not change and remain at about 45 and 55\%, respectively (Figures 1B-E and S1).

Thus, the structural differences between P. leiognathi and V. harveyi luciferases affect the fluorescent properties of these highly homologous proteins only in the native state when the globules are the most densely packed.

\subsection{Comparison of the Stability of the Luciferases Structures}

To reveal the unfolding stages of the two luciferases, we used time-resolved fluorescence spectroscopy in combination with other traditional techniques (circular dichroism spectroscopy and steady-state fluorescence). Measuring various optical characteristics of the proteins at different urea concentrations made it possible to determine the thermodynamic parameters of the unfolding luciferases (Table 1) and to plot a diagram of the relative position of the transitions during denaturation with the corresponding thermodynamic characteristic $\Delta \mathrm{G}_{\mathrm{H} 2 \mathrm{O}}$ (Figure 4).

As seen from Figure 4, the transitions of the luciferases demonstrated a similar width, except for the first transition determined by $\tau_{1}$. The difference in the transition widths and midpoints causes the obtained relationship between the free energy changes $\left(\Delta \mathrm{G}_{\mathrm{H} 2 \mathrm{O}}\right.$, Table 1) for the two luciferases. In spite of the shift in the transition midpoint for the higher urea concentration, $P$. leiognathi luciferase displayed a lower $\Delta \mathrm{G}_{\mathrm{H} 2 \mathrm{O}}$ during the first transition, determined by $\tau_{1}$. The change in the time-resolved characteristics of the protein fluorescence points out that the P. leiognathi luciferase has a more stable tertiary and secondary structure than the $V$. harveyi luciferase, i.e., the transitions have higher energetic barriers $\left(\tau_{1}\right.$, Figure 4$)$. Of note, the opposite result obtained by $\tau_{1}$ for the initial denaturation stage is in a good agreement with the conclusions made in [29]. Based on the thermal inactivation of the luciferases from different bacterial species, Holzman et al. observed that the apparent melting point of the $\mathrm{V}$. harveyi luciferase is at least $10^{\circ} \mathrm{C}$ higher than that of the P. phosphoreum luciferase (which is of the same subfamily with the P. leiognathi protein). This study seems to be the only one which is concerned with the indirect comparison of structural stability of the luciferases from the different bacterial species. Later, it was shown that the urea-induced loss of enzymatic activity of the $V$. harveyi luciferase occurs with a midpoint at about $1.6 \mathrm{M}$ of urea [30], indicating that it corresponds to the first structural transition observed with spectroscopic methods. 


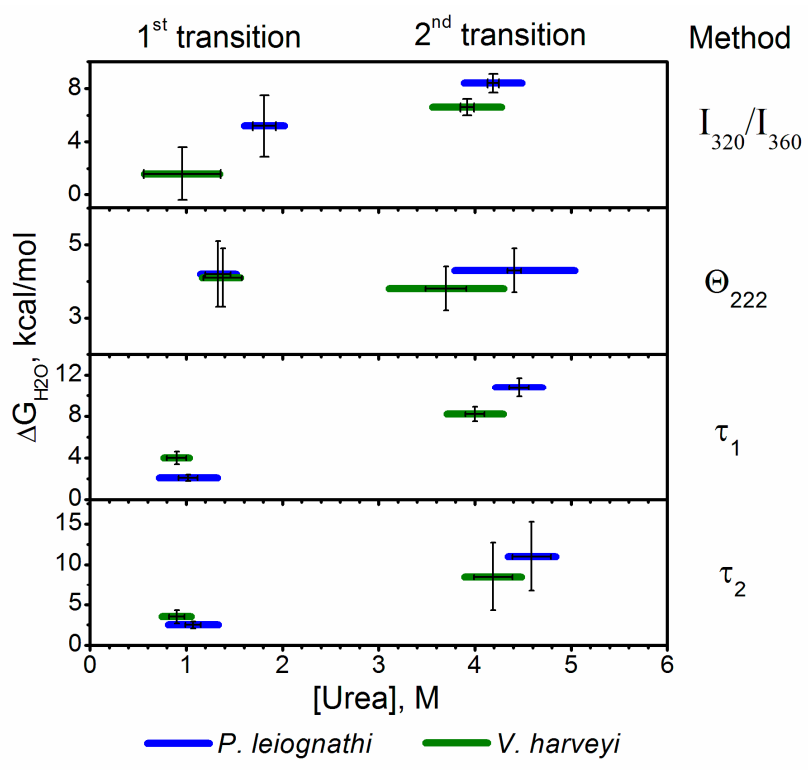

Figure 4. The urea concentration ranges of the structural transitions for the luciferases from $V$. harvey (green) and P. leiognathi (blue) and corresponding $\Delta \mathrm{G}_{\mathrm{H} 2 \mathrm{O}}$, as determined by different techniques (Table 1). The length of the sticks reflects the transition width $\mathrm{k}_{\mathrm{i}}$ in accordance with the parameters obtained by fitting with a Boltzmann function; the middle of the sticks is the transition midpoint $[\text { Urea }]_{50 \%}$.

We found that the secondary structure of the $V$. harveyi luciferase demonstrates lower stability $\left(\Theta_{222}\right.$, Figure 4$)$ than the P. leiognathi luciferase. According to the $\Theta_{222}$ change, the second visible transitions start at 3.0 and $3.8 \mathrm{M}$ of urea for $V$. harveyi and P. leiognathi luciferase, respectively. The structural origins for this difference are discussed below.

Finally, considering our results in terms of the protein unfolding pathway (as the number and the structure of the intermediate species), we can conclude that the unfolding pathway is the same for the studied luciferases. It is an important result because, in some cases, different unfolding pathways have been confirmed for highly homologous proteins (for example, alpha amylases with $88 \%$ sequence similarity [31]).

\subsection{The Origin of the Difference in Stability of Two Luciferases}

To identify the regions within the luciferase structures which could demonstrate less conformational stability, we used algorithms predicting intrinsically disordered regions relative to the approach described in [32]. This approach is based on the utilization of computational tools for the per-residue evaluation of the intrinsic disorder predisposition to search for the "weakest spot" of a query protein. We used PONDR VLXT software [33] to predict the tendency of certain regions of a polypeptide chain of the luciferases to be either structured or intrinsically disordered based on statistics or properties of amino acid residues. The analysis revealed that the $\alpha$-subunits of $V$. harveyi and P. leiognathi luciferases demonstrate very similar properties: about $22 \%$ of their amino acid residuals (a.r.) were predicted as located in disordered parts within 8 regions, with the longest one of 18 a.r. (Figure 5). However, for the $\beta$-subunits different patterns were obtained: for the $V$. harveyi luciferase about $20 \%$ of a.r. were predicted to be disordered, whereas for the P. leiognathi luciferase only about $3 \%$ of those a.r. were found. The large region of $227-325$ a.r. of the $\beta$-subunit demonstrates different predisposition to be disordered for the $V$. harveyi and P. leiognathi luciferase. In the crystal structure of the luciferase [13], the $\alpha$-helixes and $\beta$-strands were resolved in this region. The apparent contradiction could mean that this part of the subunit requires interaction with the remaining rigidly packed part of the protein to obtain the correct three-dimensional structure [32]. Thus, our analysis indicates that the observed lower stability of the secondary structure of the $V$. harveyi luciferase in comparison with the P. leiognathi enzyme could be attributed to the difference in the 
sequence of the C-terminal part of the $\beta$-subunit. The large width of the second transition, revealed by the CD technique for the $V$. harveyi luciferase $\left(\Theta_{222}\right.$, Figure 4$)$, could reflect the unfolding of the $\beta$-subunit first, which occurs at lower urea concentrations than for the $P$. leiognathi enzyme. Moreover, no tryptophan residues are located in the presumably weakened region of the luciferase $\beta$-subunit, so the fluorescent signal is not sensitive to the initial stage of the second transition, in contrast to the circular dichroism signal (Figure 4).

A

$\alpha$-subunit

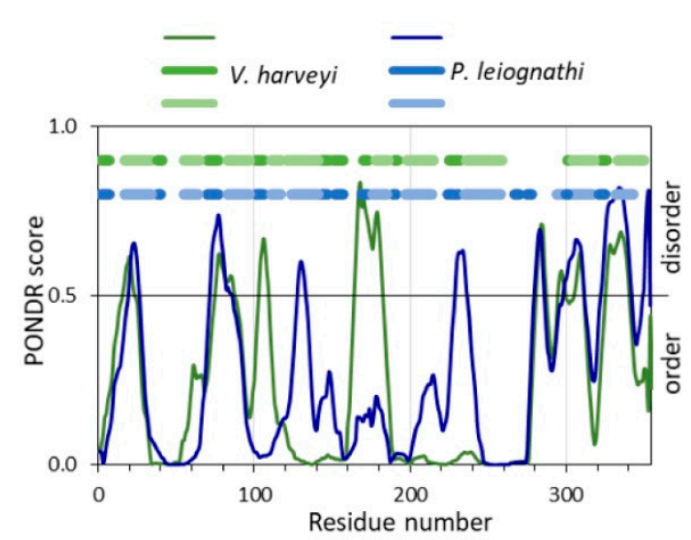

B

$\beta$-subunit

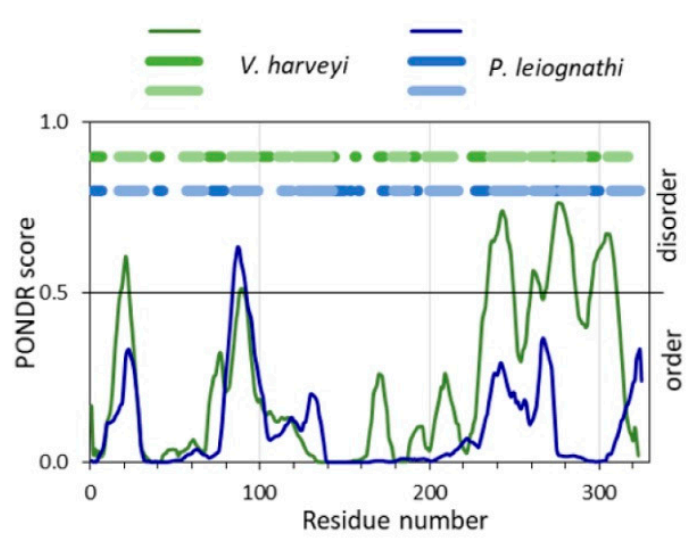

Figure 5. Intrinsic disorder propensities for amino acid sequences of the $\alpha$-subunit (A) and $\beta$-subunit (B) of the luciferases $V$. harveyi (green lines) and P. leiognathi (blue lines), as predicted with PONDR FIT [33]. Horizontal thick lines refer to the position of the amino acids, which are included in an $\alpha$-helix (lighter color) or $\beta$-strand (darker color). Distribution of secondary structure segments in polypeptide chains was retrieved using UCSF Chimera software.

\subsection{Informativeness of Different Experimental Techniques on Luciferase Unfolding}

The results obtained in this work made it possible not only to compare the stability of the secondary and tertiary structures of the closely related proteins, but also to evaluate the sensitivity of different experimental methods to individual stages of protein unfolding. Thus, the first stage of the unfolding of both luciferases has little effect on the position of their fluorescence spectrum $\left(\mathrm{I}_{320} / \mathrm{I}_{360}\right)$. This parameter changes by less than $20 \%$, while the shifts in other optical parameters are more pronounced, as can be seen from the values of the fraction in Table 1. This can be explained by the fact that the unfolding of the C-terminal domain of proteins is probably not accompanied by a significant change in the polarity of the environment of the majority of the tryptophan residues. The penetration of water into the deeper parts of the luciferases occurs during the next unfolding stages. Thus, in this study, the time-resolved fluorescence demonstrates obvious advantages and detects the subtle initial structural transitions of the proteins, which cannot be seen from the steady-state emission spectra.

It also turned out to be impossible to reliably obtain the characteristics of the second transition of the luciferases with a midpoint of about $4 \mathrm{M}$ of urea using lifetime component $\tau_{2}$. The amplitude of the whole change of $\tau_{2}$ during this unfolding is only $\sim 0.1 \mathrm{~ns}$, and the resolution of the instrument does not allow for accurate determination of the gradual shift of this lifetime component.

For the fluorescence of bacterial luciferase, lifetime components $\tau_{1}$ and $\tau_{2}$ were found to reflect similar information about the stages of protein unfolding (Table 1). It was previously shown that this is not the case for some proteins (see the example of bovine carboanhydrase II [21]). It should be emphasized that the structural and/or dynamic reasons for the similarities or differences in information based on the fluorescence lifetimes of the protein are not yet understood. The study of denaturation of various proteins containing a single tryptophan in their structure could shed light on this problem. The recent findings 
point out the importance of the distribution of electrostatic potential around tryptophan in modulating the probability of the formation of a charge transfer state after photon absorption, which, in turn, influences the quantum yield and the lifetime of the tryptophan fluorescence [34]. However, an unambiguous and available method for analyzing this structural characteristic for a specific protein has not yet been developed.

\section{Materials and Methods}

\subsection{Materials}

Lyophilized recombinant luciferase from Photobacterium leiognathi (99\% purity) was purchased from Biolumdiagnostika Ltd. (Krasnoyarsk, Russia). Urea (Panreac, Germany), $\mathrm{Na}_{2} \mathrm{HPO}_{4}$ and $\mathrm{KH}_{2} \mathrm{PO}_{4}$ (AppliChem, Germany) were used without additional purification. All solutions were prepared using $\mathrm{Na}_{2} \mathrm{HPO}_{4} / \mathrm{KH}_{2} \mathrm{PO}_{4}$ buffer $(0.05 \mathrm{M}$, pH 6.9). The concentration of urea (0.25-8 M) was determined using a laboratory refractometer, IRF-454 B2M (Kazan Optical and Mechanical Plant, Russia).

\subsection{Expression and Purification of V. harveyi luciferase}

The pET19b-VhLuxAB plasmid containing the coding part of the $V$. harveyi (MAV strain) luxAB gene was expressed in E. coli strain BL21 (DE3) via Codon Plus RIPL (Stratagene, USA). The cells harboring the plasmid were grown in the LB medium containing $200 \mu \mathrm{g} / \mathrm{mL}$ ampicillin at $37^{\circ} \mathrm{C}$, and IPTG was added to the final concentration of $0.25 \mathrm{mM}$ when the cell density reached an $\mathrm{OD}_{600}$ of $0.6-0.7$. Following the induction with IPTG, the cell culture was allowed to grow for $18 \mathrm{~h}$ at $23^{\circ} \mathrm{C}$. With this expression, luciferase mostly accumulated in the cytoplasm in a soluble form. Cells were harvested, centrifuged, and resuspended in $5 \mathrm{~mL} \times g$ of $20 \mathrm{mM}$ Tris- $\mathrm{HCl}, \mathrm{pH} 7.0$ before lysis by ultrasonication. After lysis, the cell debris was removed by centrifugation, and the supernatant containing the extracted luciferase was purified with ion-exchange chromatography on a HiTrap $Q$ HP 5 column (GE Healthcare, USA) with a $\mathrm{NaCl}$ linear gradient (0-1 M). The portion of the eluate containing luciferase was concentrated, diluted 25 times in $50 \mathrm{mM}$ phosphate buffer, $\mathrm{pH} 7.0$, and sedimented with ammonium sulfate $(25.8 \mathrm{~g}$ per every $50 \mathrm{~mL}$ of the solution). After centrifugation the precipitate was diluted in $50 \mathrm{mM}$ phosphate buffer, $\mathrm{pH}$ 7.0 , with $0.15 \mathrm{M} \mathrm{NaCl}$ and purified with gel filtration on a Superdex 75 (GE Healthcare, USA). Protein concentrations were first estimated using the DC Protein Assay Kit with bovine serum albumin as a standard (Bio-Rad, USA). More accurate protein concentration was determined spectrophotometrically using the protein extinction coefficient calculated by the method of Gill and von Hippel [35].

To confirm the proper luciferase folding, its functionality was tested by the flavininjection technique [36].

\subsection{Steady-State Fluorescence}

The spectral characteristics of the luciferases at concentration of $1-2 \mu \mathrm{M}$ were measured after $18 \mathrm{~h}$ of incubation with urea $(0.25-8 \mathrm{M})$ at room temperature. The measurements were carried out at $25^{\circ} \mathrm{C}$ unless otherwise specified.

The absorption spectra were measured using a Cary 5000 spectrophotometer (Agilent Technologies, Australia) with an integrated Pelletier temperature controller. Steady-state fluorescence spectra of the protein were measured with a Fluorolog-3 spectrofluorometer (Horiba Jobin Yvon, USA). The intensity was collected within the range of 305-450 nm under excitation at $296 \mathrm{~nm}$. All fluorescence spectra were corrected for PMT spectral sensitivity, inner filter effect and background signal [28].

The spectral shift of fluorescence was described in terms of the ratio of the intensities at 320 and $360 \mathrm{~nm}, \mathrm{I}_{320} / \mathrm{I}_{360}$.

\subsection{Time-Resolved Fluorescence}

Time-resolved fluorescence decays were obtained using a time-correlated single photon counting (TCSPC) DeltaHub module of Fluorolog-3 (Horiba Jobin Yvon, USA). A 
NanoLED N-295 with the peak wavelength at $296 \mathrm{~nm}$ and pulse duration of $<1.2 \mathrm{~ns}$ was applied for excitation. Emission was detected within the range of $320-410 \mathrm{~nm}$ with a step of $5 \mathrm{~nm}$; time resolution was $0.027 \mathrm{~ns} /$ channel.

Time-resolved fluorescence experiments were carried out using the TCSPC technique [37]. The fluorescence lifetime profile of the protein consisting of a sum of three exponentials with lifetimes $\tau_{i}$ and amplitudes $\alpha_{i}$ was deconvoluted from the observed fluorescence decay $I(t)$ :

$$
I(t)=S(t) \otimes \sum_{i=1}^{N} \alpha_{i} e^{-t / \tau_{i}}
$$

where $S(t)$ is the instrumental response function measured with a highly scattering solution, Ludox. The fit quality was evaluated by its global $\chi^{2}$ value and weighted residuals.

The decay-associated spectra of fluorescence were calculated as follows:

$$
I_{i}^{\lambda}=f_{i}^{\lambda} I_{S S}
$$

where $I_{s s}$ is the steady-state fluorescence spectra, and $f_{i}^{\lambda}$ is the contribution of each lifetime component to fluorescence at a certain wavelength $\lambda$ :

$$
f_{i}^{\lambda}=\frac{\alpha_{i}^{\lambda} \tau_{i}}{\sum_{j=1}^{N} \alpha_{j}^{\lambda} \tau_{j}}
$$

where $\alpha_{i}^{\lambda}$ is the amplitude of the $i$-component of the lifetime at a wavelength $\lambda$. The total spectral contribution of the lifetime component $\tau_{i}$ to protein fluorescence, $f_{i}$, was calculated as the average of the $f_{i}^{\lambda}$ values.

\subsection{Circular Dichroism Spectroscopy}

The CD spectra were obtained using Jasco- 810 and a Jasco- 1500 spectropolarimeters (Jasco, Japan). The far-UV CD spectra were recorded for P. leiognathi luciferase in a $1 \mathrm{~mm}$ path length cell in the range of 190-260 nm, with a step size of $0.2 \mathrm{~nm}$, and for $V$. harveyi luciferase in a $0.1 \mathrm{~mm}$ path length cell in the range of $190-250 \mathrm{~nm}$, with a step size of 1 $\mathrm{nm}$. At least two scans were averaged for all spectra. Spectra were baseline corrected. The measurements were performed at room temperature.

\subsection{Data Treatment}

The urea-induced transition curves obtained from the change of the optical parameters were fitted by the double-Boltzmann function, available in the Origin 8.0 software (OriginLab Corp.). The transition width, $\mathrm{k}_{\mathrm{i}}$, and the transition midpoints, [Urea $]_{50 \%}$, were obtained to compare the structural stability of the proteins.

\subsection{Förster Resonance Energy Transfer (FRET)}

Efficiency of the non-radiative energy transfer between tryptophan residues via the Förster mechanism [38] was evaluated as:

$$
E=\frac{1}{1+\frac{2 / 3}{\kappa^{2}}\left(\frac{R}{R_{0}}\right)^{6}}
$$

where $R_{0}$ is the Förster distance, $R$ is the distance between the geometric centers of the indole rings of the donor and the acceptor, and $\kappa^{2}$ is the factor of mutual orientation of the donor and the acceptor.

Orientation factor was determined in accordance with:

$$
\kappa^{2}=\left(\cos \theta-3 \cos \theta_{\mathrm{A}} \cos \theta_{\mathrm{D}}\right)^{2}
$$


where $\theta$ is the angle between the directions of the emission oscillator of the donor and the absorption oscillator of the acceptor; $\theta_{\mathrm{A}}$ and $\theta_{\mathrm{D}}$ are the angles between the oscillators and the vector connecting the geometric centers of the donor and acceptor molecules [28].

The calculations were performed under the assumption of rigid oscillators. $R$ and $\kappa^{2}$ were calculated based on the atom coordinates. Due to the uncertainty of the donor quantum yield and the overlap integral value of the donor fluorescence and the acceptor absorption, $E$ for the Trp-Trp energy transfer was calculated using $R_{0}=7.8 \AA$ [26].

\subsection{Molecular Dynamics of the Proteins}

Molecular dynamics (MD) simulations were performed for the three-dimensional structures of the $V$. harveyi and P. leiognathi luciferases. The crystal structure of $V$. harveyi was downloaded from Protein Data Bank (PDB ID: 3FGC) in complex with FMN [13]. Initial coordinates of $P$. leiognathi luciferase were obtained by homology-based modeling using the SWISS-MODEL server and the crystal structure of $V$. harveyi luciferase as a template [39]. The sequence of $P$. leiognathi luciferases was retrieved from the UniProt database, including the $\alpha$-subunit (P09140) and $\beta$-subunit (P09141) [40]. The high identity between the target and the template sequences ( 54.08 and $45.45 \%$ for $\alpha$ and $\beta$-subunits) ensured a high quality of the P. leiognathi luciferase three-dimensional model (GMQE $=0.78$, QMEAN $=-1.02)$ [9].

The missed disordered segment of the mobile loop of the $\alpha$-subunit (283-290 amino acid residues) in the $V$. harveyi crystal was reconstructed by MODELLER [41]. Before starting the energy minimization step, FMN was removed from the 3FGC structure. MD simulations were conducted using GROMACS 5. 1. 4 with the CHARMM36 force field and the TIP3P explicit water. The following simulation protocol was the same for each protein [42-44]. The protein was centered in a cubic box with periodic boundary conditions at least $12 \AA$ away from each of the box edges and solvated with water molecules. The net charge of the system was neutralized with sodium ions. Complete information about the system size is provided in Table S1.

The system was minimized with the steepest descent method (maximum force of $1000.0 \mathrm{~kJ} / \mathrm{mol}$ ). The cutoff distance for the short-range non-bonded interactions was $12 \AA$, and the electrostatic interactions were treated using the particle mesh Ewald method. An integration step of $2.0 \mathrm{fs}$ was used, and bonds were constrained with the LINCS algorithm. The minimized system was heated and equilibrated at $300 \mathrm{~K}$ for $5 \mathrm{~ns}$ in the NVT ensemble with the restrained protein. The following NPT equilibrations were carried out in two stages. In the first phase, a $10 \mathrm{~ns}$ equilibration was performed with the restraint protein heavy atoms. In the second, a $10 \mathrm{~ns}$ equilibration followed, and all atoms in the system were free to move.

Production MD simulations were carried out in the NPT ensemble for $40 \mathrm{~ns}$ for each luciferase, collecting coordinates of the system every $10 \mathrm{ps.} \mathrm{A} \mathrm{modified} \mathrm{Berendsen}$ (V-rescale) thermostat and a Parrinello-Rahman barostat were employed.

Reconstruction of the movable loop segment in the 3FGC crystal, all visualizations and tryptophan alignment were performed using the UCSF CHIMERA software package [45]. The positions of amino acids in the luciferase structure is indicated in accordance with the sequence of $V$. harveyi luciferase.

The distance distributions between the mass centers of the two tryptophan indole rings were calculated using the gmx distance plugin in GROMACS. PONDR-VLXT was used to estimate the intrinsic disorders of both luciferase structures [33].

\section{Conclusions}

The present study has been carried out in the field of molecular biophysics and was directed at identifying factors affecting the conformational stability of proteins, as well as at developing methods of assessment and comparison of their structural stability. We have investigated the change of spectroscopic properties during urea-induced unfolding of two homologous proteins $-V$. harveyi and $P$. leiognathi luciferases-with special attention to the time-resolved fluorescence of these proteins. Despite the high percentage of identical and 
similar amino acids in their sequences, the studied enzymes belong to different subfamilies of bacterial luciferases with differing functionality, as previously shown by phylogenetic analysis. The results of our study indicate that the "fast" P. leiognathi luciferase has more stable tertiary and secondary structures than the "slow" V. harveyi luciferase. The most remarkable differences between the luciferases were revealed by time-resolved fluorescence in the range of $0-2 \mathrm{M}$ of urea. We have analyzed the positions and the microenvironment of the tryptophan residues within the luciferase globule and found that in the $V$. harveyi protein they are packed more tightly. This enhances short-range interactions and aggravates quenching processes, leading to a decrease in the fluorescence lifetime. During the first stage of the urea-induced denaturation, both luciferases acquire the conformation of an inactive dimer, in which the quenching effects are removed and the difference between the proteins disappears. Our study has revealed that the tryptophans located in or nearby the C-terminal domain of the protein $\alpha$-subunit ( $\alpha \mathrm{W} 194, \alpha \mathrm{W} 250, \alpha \mathrm{W} 277)$ make the most significant contribution to the observed individual fluorescent properties of the bacterial luciferase. Meanwhile, the difference in the secondary structure stability could be due to the peculiarities of the sequence of the protein $\beta$-subunit. Previously, we demonstrated for carboanhydrase II that the two lifetimes of the protein fluorescence could probe different structural transitions, i.e., different stages of protein unfolding. In this work, we found for the bacterial luciferases that both fluorescence lifetimes report about the same structural transitions, but only using time-resolved parameters makes it possible to identify the difference in conformational stability of the two homologous proteins. However, to answer the question of whether the found properties of conformational stability is a sign of the entire subfamily ("fast" or "slow" luciferases) or is an individual feature of only the studied enzymes, further research is required.

Supplementary Materials: The following are available online at https:/ /www.mdpi.com/article/10 .3390/ijms221910449/s1.

Author Contributions: Conceptualization, B.S.M., E.V.N.; methodology, M.A.G., B.S.M. and E.V.N.; investigation, D.V.G., L.A.S., N.E.K., L.P.B. and E.V.N.; writing-original draft preparation, D.V.G., L.A.S., L.P.B. and E.V.N.; writing-review and editing, D.V.G., M.A.G., L.A.S., L.P.B., N.E.K., B.S.M., V.A.K. and E.V.N.; supervision, V.A.K., E.V.N.; funding acquisition, D.V.G., E.V.N. and V.A.K. All authors have read and agreed to the published version of the manuscript.

Funding: The research was partially funded by the Ministry of Science and Higher Education of the Russian Federation (projects No. FSRZ-2020-0006); by the RFBR and Krasnoyarsk Territory and Krasnoyarsk Regional Fund of Science (projects No. 20-44-243002 and 20-44-240006); and by the RFBR (project No. 20-34-90118).

Institutional Review Board Statement: Not applicable.

Informed Consent Statement: Not applicable.

Data Availability Statement: The data presented in this study are available on request from the corresponding author.

Acknowledgments: The authors are grateful to Olesya Lashchuk for assistance in time-resolved experiments, to Anvar Ismailov (Lomonosov Moscow State University) for the generous provision of $V$. harveyi bacteria and to Anna Deeva (Siberian Federal University) for the help in the preparation of the manuscript.

Conflicts of Interest: The authors declare no conflict of interest.

\section{References}

1. Blancas-Mejía, L.M.; Ramirez-Alvarado, M. Systemic amyloidoses. Annu. Rev. Biochem. 2013, 82, 745-774. [CrossRef] [PubMed]

2. Stefani, M.; Dobson, C.M. Protein aggregation and aggregate toxicity: New insights into protein folding, misfolding diseases and biological evolution. J. Mol. Med. 2003, 81, 678-699. [CrossRef] [PubMed]

3. Lakowicz, J.R. (Ed.) Topics in Fluorescence Spectroscopy: Protein Fluorescence; Kluwer Academic/Plenum: New York, NY, USA, 2000; Volume 6. 
4. Krishnamoorthy, G. Fluorescence spectroscopy for revealing mechanisms in biology: Strengths and pitfalls. J. Biosci. 2018, 43, 555-567. [CrossRef]

5. Tinikul, R.; Chunthaboon, P.; Phonbuppha, J.; Paladkong, T. Bacterial luciferase: Molecular mechanisms and applications. Enzymes 2020, 47, 427-455.

6. Gregor, C.; Gwosch, K.C.; Sahl, S.J.; Hell, S.W. Strongly enhanced bacterial bioluminescence with the ilux operon for single-cell imaging. Proc. Natl. Acad. Sci. USA 2018, 115, 962-967. [CrossRef] [PubMed]

7. Kratasyuk, V.A.; Esimbekova, E.N. Applications of luminous bacteria enzymes in toxicology. Comb. Chem. High Throughput Screen. 2015, 18, 952-959. [CrossRef]

8. Gui, Q.; Lawson, T.; Shan, S.; Yan, L.; Liu, Y. The application of whole cell-based biosensors for use in environmental analysis and in medical diagnostics. Sensors 2017, 17, 1623. [CrossRef] [PubMed]

9. Deeva, A.A.; Temlyakova, E.A.; Sorokin, A.A.; Nemtseva, E.V.; Kratasyuk, V.A. Structural distinctions of fast and slow bacterial luciferases revealed by phylogenetic analysis. Bioinformatics 2016, 32, 3053-3057. [CrossRef]

10. Nealson, K.H.; Hastings, J.W. Bacterial bioluminescence: Its control and ecological significance. Microbiol. Rev. 1979, 43, 496-518. [CrossRef]

11. Luo, Y.; Liu, Y.J. Revisiting the origin of bacterial bioluminescence: QM/MM study on oxygenation reaction of reduced flavin in protein. Chem. Phys. Chem. 2019, 20, 405-409. [CrossRef]

12. Fisher, A.J.; Thompson, T.B.; Thoden, J.B.; Baldwin, T.O.; Rayment, I. The 1.5-Å resolution crystal structure of bacterial luciferase in low salt conditions. J. Biol. Chem. 1996, 271, 21956-21968. [CrossRef]

13. Campbell, Z.T.; Weichsel, A.; Montfort, W.R.; Baldwin, T.O. Crystal structure of the bacterial luciferase/flavin complex provides insight into the function of the $\beta$ subunit. Biochemistry 2009, 48, 6085-6094. [CrossRef]

14. Apuy, J.L.; Park, Z.Y.; Swartz, P.D.; Dangott, L.J.; Russell, D.H.; Baldwin, T.O. Pulsed-alkylation mass spectrometry for the study of protein folding and dynamics: Development and application to the study of a folding/unfolding intermediate of bacterial luciferase. Biochemistry 2001, 40, 15153-15163. [CrossRef]

15. Campbell, Z.T.; Baldwin, T.O. Two lysine residues in the bacterial luciferase mobile loop stabilize reaction intermediates. J. Biol. Chem. 2009, 284, 32827-32834. [CrossRef]

16. Baldwin, T.O.; Ziegler, M.M.; Powers, D.A. Covalent structure of subunits of bacterial luciferase: NH2-terminal sequence demonstrates subunit homology. Proc. Natl. Acad. Sci. USA 1979, 76, 4887-4889. [CrossRef]

17. Sparks, J.M.; Baldwin, T.O. Functional implications of the unstructured loop in the $(\beta / \alpha)_{8}$ barrel structure of the bacterial luciferase a subunit. Biochemistry 2001, 40, 15436-15443. [CrossRef] [PubMed]

18. Thoden, J.B.; Holden, H.M.; Fisher, A.J.; Sinclair, J.F.; Wesenberg, G.; Baldwin, T.O.; Rayment, I. Structure of the $\beta_{2}$ homodimer of bacterial luciferase from Vibrio harveyi: X-ray analysis of a kinetic protein folding trap. Protein Sci. 1997, 6, 13-23. [CrossRef]

19. Xin, X.; Xi, L.; Tu, S.C. Functional consequences of site-directed mutation of conserved histidyl residues of the bacterial luciferase. alpha. subunit. Biochemistry 1991, 30, 11255-11262. [CrossRef] [PubMed]

20. Inlow, J.K.; Baldwin, T.O. Mutational analysis of the subunit interface of Vibrio harveyi bacterial luciferase. Biochemistry 2002, 41, 3906-3915. [CrossRef]

21. Nemtseva, E.V.; Gerasimova, M.A.; Melnik, T.N.; Melnik, B.S. Experimental approach to study the effect of mutations on the protein folding pathway. PLoS ONE 2019, 14, e0210361. [CrossRef] [PubMed]

22. Kuznetsova, I.; Yakusheva, T.; Turoverov, K. Contribution of separate tryptophan residues to intrinsic fluorescence of actin. Analysis of 3D structure. FEBS Lett. 1999, 452, 205-210. [CrossRef]

23. Noland, B.W.; Dangott, L.J.; Baldwin, T.O. Folding, stability, and physical properties of the $\alpha$ subunit of bacterial luciferase. Biochemistry 1999, 38, 16136-16145. [CrossRef] [PubMed]

24. Ghisaidoobe, A.B.; Chung, S.J. Intrinsic tryptophan fluorescence in the detection and analysis of proteins: A focus on Förster resonance energy transfer techniques. Int. J. Mol. Sci. 2014, 15, 22518-22538. [CrossRef] [PubMed]

25. Li, Z.; Valkova, N.; Meighen, E. Spectral properties of Trp182, Trp194, and Trp250 on the $\alpha$ subunit of bacterial luciferase. Biochem. Biophys. Res. Commun. 1999, 263, 820-824. [CrossRef]

26. Eisinger, J.; Feuer, B.; Lamola, A.A. Intramolecular singlet excitation transfer. Applications to polypeptides. Biochemistry 1969, 8 , 3908-3915. [CrossRef] [PubMed]

27. Chen, Y.; Barkley, M.D. Toward understanding tryptophan fluorescence in proteins. Biochemistry 1998, 37, 9976-9982. [CrossRef] [PubMed]

28. Lakowicz, J. Principles of Fluorescence Spectroscopy, 3rd ed.; Springer Science \& Business Media: New York, NY, USA, 2006.

29. Holzman, T.F.; Baldwin, T.O. The effects of phosphate on the structure and stability of the luciferases from Beneckea harveyi, Photobacterium, fischeri, and, Photobacterium phosphoreum. Biochem. Biophys. Res. Commun. 1980, 94, 1199-1206. [CrossRef]

30. Clark, A.; Sinclair, J.; Baldwin, T. Folding of bacterial luciferase involves a non-native heterodimeric intermediate in equilibrium with the native enzyme and the unfolded subunits. J. Biol. Chem. 1993, 268, 10773-10779. [CrossRef]

31. Ahmad, A.; Mishra, R. Different unfolding pathways of homologous alpha amylases from Bacillus licheniformis (BLA) and Bacillus amyloliquefaciens (BAA) in GdmCl and urea. Int. J. Biol. Macromol. 2020, 159, 667-674. [CrossRef]

32. Nagibina, G.S.; Glukhova, K.A.; Uversky, V.N.; Melnik, T.N.; Melnik, B.S. Intrinsic disorder-based design of stable globular proteins. Biomolecules 2020, 10, 64. [CrossRef] 
33. Romero, P.; Obradovic, Z.; Li, X.; Garner, E.C.; Brown, C.J.; Dunker, A.K. Sequence complexity of disordered protein. Proteins Struct. Funct. Bioinform. 2001, 42, 38-48. [CrossRef]

34. Callis, P.R.; Vivian, J.T. Understanding the variable fluorescence quantum yield of tryptophan in proteins using QM-MM simulations. Quenching by charge transfer to the peptide backbone. Chem. Phys. Lett. 2003, 369, 409-414. [CrossRef]

35. Gill, S.C.; Von Hippel, P.H. Calculation of protein extinction coefficients from amino acid sequence data. Anal. Biochem. 1989, 182, 319-326. [CrossRef]

36. Baldwin, T.O.; Ziegler, M.M.; Green, V.A.; Thomas, M.D. Overexpression of bacterial luciferase and purification from recombinant sources. Methods Enzymol. Acad. Press 2000, 305, 135-152.

37. O'Connor, D. Time-Correlated Single Photon Counting; Academic Press: Cambridge, MA, USA, 2012.

38. Förster, T. Transfer mechanisms of electronic excitation energy. Radiat. Res. Suppl. 1960, 2, 326-339. [CrossRef]

39. Waterhouse, A.; Bertoni, M.; Bienert, S.; Studer, G.; Tauriello, G.; Gumienny, R.; Heer, F.T.; de Beer, T.A.P.; Rempfer, C.; Bordoli, L.; et al. SWISS-MODEL: Homology modelling of protein structures and complexes. Nucleic Acids Res. 2018, 46, W296-W303. [CrossRef]

40. The UniProt Consortium. UniProt: The universal protein knowledgebase in 2021. Nucleic Acids Res. 2021, 49, D480-D489. [CrossRef]

41. Šali, A.; Blundell, T.L. Comparative protein modelling by satisfaction of spatial restraints. J. Mol. Biol. 1993, $234,779-815$. [CrossRef]

42. Van Der Spoel, D.; Lindahl, E.; Hess, B.; Groenhof, G.; Mark, A.E.; Berendsen, H.J. GROMACS: Fast, flexible, and free. J. Comput. Chem. 2005, 26, 1701-1718. [CrossRef]

43. Best, R.B.; Zhu, X.; Shim, J.; Lopes, P.E.; Mittal, J.; Feig, M.; MacKerell, A.D., Jr. Optimization of the additive CHARMM all-atom protein force field targeting improved sampling of the backbone $\mathrm{f}, \mathrm{y}$ and side-chain $\mathrm{c} 1$ and $\mathrm{c} 2$ dihedral angles. J. Chem. Theory Comput. 2012, 8, 3257-3273. [CrossRef]

44. Jorgensen, W.L.; Chandrasekhar, J.; Madura, J.D.; Impey, R.W.; Klein, M.L. Comparison of simple potential functions for simulating liquid water. J. Chem. Phys. 1983, 79, 926-935. [CrossRef]

45. Pettersen, E.F.; Goddard, T.D.; Huang, C.C.; Couch, G.S.; Greenblatt, D.M.; Meng, E.C.; Ferrin, T.E. UCSF Chimera-A visualization system for exploratory research and analysis. J. Comput. Chem. 2004, 25, 1605-1612. [CrossRef] [PubMed] 